\title{
Dietary patterns during pregnancy and the risk of postpartum depression: the mother-child 'Rhea' cohort in Crete, Greece
}

Leda Chatzi ${ }^{1, *}$, Vasiliki Melaki ${ }^{1}$, Katerina Sarri ${ }^{1}$, loanna Apostolaki ${ }^{1}$, Theano Roumeliotaki ${ }^{1}$, Vaggelis Georgiou ${ }^{1}$, Maria Vassilaki ${ }^{1}$, Antonis Koutis ${ }^{1}$, Panos Bitsios ${ }^{2}$ and Manolis Kogevinas $3,4,5$

'Department of Social Medicine, Faculty of Medicine, University of Crete, PO Box 2208, Heraklion 71003, Crete, Greece: ${ }^{2}$ Faculty of Medicine, Department of Psychiatry and Behavioral Sciences, University of Crete, Heraklion, Greece: ${ }^{3}$ Centre for Research in Environmental Epidemiology (CREAL), Barcelona, Spain: ${ }^{4} \mathrm{CIBER}$, Epidemiologia y Salud Publica, Barcelona, Spain: ${ }^{5}$ National School of Public Health, Athens, Greece

Submitted 18 August 2010: Accepted 23 November 2010: First published online 11 April 2011

\begin{abstract}
Objective: To identify and describe dietary patterns in a cohort of pregnant women, and investigate whether dietary patterns during pregnancy are related to postpartum depression (PPD).

Design: The study uses data from the prospective mother-child cohort 'Rhea' study. Pregnant women completed an FFQ in mid-pregnancy and the Edinburg Postpartum Depression Scale (EPDS) at 8-10 weeks postpartum. Dietary patterns during pregnancy ('health conscious', 'Western') were identified using principal component analysis. Associations between dietary patterns categorized in tertiles and PPD symptoms were investigated by multivariable regression models after adjusting for confounders.

Setting: Heraklion, Crete, Greece, 2007-2010.

Subjects: A total of 529 women, participating in the 'Rhea' cohort.

Results: High adherence to a 'health conscious' diet, characterized by vegetables, fruit, pulses, nuts, dairy products, fish and olive oil, was associated with lower EPDS scores (highest $v$. lowest tertile: $\beta$-coefficient $=-1 \cdot 75, P=0 \cdot 02$ ). Women in the second (relative risk $(\mathrm{RR})=0 \cdot 52,95 \% \mathrm{CI} 0 \cdot 30,0 \cdot 92)$ or third tertile $(\mathrm{RR}=0 \cdot 51$, $95 \% \mathrm{CI} 0 \cdot 25,1 \cdot 05$ ) of the 'health conscious' dietary pattern were about $50 \%$ less likely to have high levels of PPD symptoms (EPDS $\geq 13$ ) compared with those in the lowest tertile.

Conclusions: This is the first prospective study showing that a healthy diet during pregnancy is associated with reduced risk for PPD. Additional longitudinal studies and trials are needed to confirm these findings.
\end{abstract}

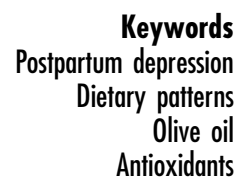

Postpartum depression (PPD) is defined as a major depressive disorder with a specifier of postpartum onset within 1 month after childbirth ${ }^{(1)}$. Approximately 12-16\% of women experience PPD worldwide ${ }^{(2,3)}$. These are probably conservative estimates, as cases of maternal depression are under-reported or underdiagnosed ${ }^{(4)}$. The prevalence of PPD symptomatology has been estimated from $12 \%$ to $20 \%$ in Greece, according to the Edinburg Postpartum Depression Scale (EPDS) ${ }^{(5-7)}$. The deleterious effects of PPD on offspring's development and cognitive and social functioning are well documented ${ }^{(8,9)}$.

The causes of PPD remain unclear, but nutrition, which modulates several associated factors (i.e. hormonal, immunological and biochemical), may have a plausible impact on the development and course of this complex multifactorial illness ${ }^{(10)}$. Nutritional requirements are increased during pregnancy and lactation, so that fetal and infant growth as well as maternal metabolic needs specific to reproduction are adequately supported. Randomized controlled trials, cohort and ecological studies have revealed a positive association between low levels of $n-3$ fatty acids and a higher incidence of PPD ${ }^{(11)}$. However, these studies may fail to account for the interactions between nutrients, and they do not take into consideration the fact that some nutrients are intercorrelated $^{(12)}$. Thus, interest has shifted to the study of food groups and, more recently, to dietary patterns that represent a broader picture of food and nutrient consumption and may therefore be more predictive of disease risk. The investigation of the association between dietary patterns and mental illness is still in its infancy and psychiatry lacks evidence-based primary prevention and 
treatment strategies based on dietary modification. To date, there is only one recent study that showed a protective effect of a 'healthy' dietary pattern in the high prevalence of depressive and anxiety disorders in nonpregnant Australian women ${ }^{(13)}$. However, because of its cross-sectional nature, reverse causality and confounding could not be ruled out.

The objectives of the present epidemiological study were to identify and describe dietary patterns in a population-based cohort of pregnant women in Crete, Greece, and determine prospectively the association between habitual dietary patterns during pregnancy and the risk for PPD. We hypothesized that a better diet quality would be associated with lower risk for PPD.

\section{Methods}

\section{Study sample}

The 'Rhea' project is a large mother-child study that examines prospectively a population-based cohort of pregnant women and their children at the prefecture of Heraklion, Crete ${ }^{(14)}$. Female residents (Greek and immigrants) who had become pregnant during the 12-month period starting from February 2007 were contacted and asked to participate in the study. The first contact was made at the time of the first major ultrasound examination, around week 15 of gestation or before. Women were then contacted at the 6th month of pregnancy, at birth, 8-10 weeks after delivery, 6 months after delivery, and are currently contacted 2 years after delivery. Face-to-face structured questionnaires together with self-administered questionnaires and medical records were used to obtain information on several dietary, environmental and psychosocial exposures during pregnancy and early childhood. The study was approved by the ethical committee of the University Hospital in Heraklion, Crete, Greece, and all participants provided written, informed consent after complete description of the study.

During the study recruitment period (February 2007February 2008), 1765 eligible women were approached, of whom 1610 (91\%) agreed to participate and 1317 (82\%) were followed up until delivery. Only singleton pregnancies were included in the present analysis ( $n$ 1281). A total of 726 participants provided complete questionnaire data on PPD and diet during pregnancy $(57 \%)$. Women who experienced spontaneous or induced abortions ( $n 51)$ or gave birth to stillborn infants ( $n$ 2) were excluded, as were those women with incomplete diagnostic information ( $n$ 60) for the following reasons: change of address or delivery outside Heraklion prefecture and/or incomplete or missing medical records. We also excluded twenty women with a diagnosis of depression or other psychiatric disorders before pregnancy, since these disorders are associated with a higher probability of PPD. Sixty-four additional women were excluded from the analysis because of implausible values for total energy intake (outside the range of 4184$\left.16736 \mathrm{~kJ} / \mathrm{d}(1000-4000 \mathrm{kcal} / \mathrm{d})^{(15)}\right)$. Hence, a cohort of 529 women were available for the present analysis.

\section{Depressive symptoms}

The EPDS was completed by the mothers at 8-10 weeks after delivery. The scale is a widely used ten-item rating scale designed to screen for PPD in community samples ${ }^{(16)}$. Each item is scored on a 4-point scale (from 0 to 3) and the total score ranges from 0 to 30 . The EPDS rates the intensity of depressive symptoms present within the previous $7 \mathrm{~d}$, and is designed to exclude symptoms ascribable to the somatic effects of childbirth. The Greek version of the scale was validated in $2008^{(7)}$. Scores on the EPDS can be categorized by well-validated cut-off points; in the present analysis we decided to use the cut-off point of 13 , as it has been shown to achieve high sensitivity (95\%) and specificity (93\%) when compared with clinical psychiatric interview ${ }^{(17)}$, and this score is recommended for use when reporting on probable major depression in postpartum women ${ }^{(7,18,19)}$. Therefore, in the present analysis, EPDS was used as a continuous variable, and as a categorical variable with $E P D S \geq 13$ indicating high levels of postpartum depressive symptoms.

\section{Dietary assessment during pregnancy}

The 'Rhea' FFQ was administered in mid-pregnancy (14th-18th week of gestation) assessing dietary habits over pregnancy. This is a semi-quantitative questionnaire, containing 250 food items with the following components: food frequency, dietary supplements, information on organic food consumption, different types of vegetarianism, dietary changes due to pregnancy, as well as conditions of pregnancy that could affect diet and food intake (nausea, vomiting, etc.). The questions were hierarchical, from general to more specific questions. The 250 food items recorded in the FFQ were aggregated into seventeen food groups ('cereals and cereal products', 'meat and meat products', 'fish and seafood', 'dairy products', 'eggs', 'vegetable-animal fats except olive oil', 'olive oil', 'potatoes and other starchy roots', 'pulses', 'vegetables', 'nuts', 'fruits', 'sweets and deserts', 'nonalcoholic beverages', 'alcoholic beverages', 'salty snacks' and 'miscellaneous'). For each food item, participants were asked about both frequency of consumption and average portion size. The frequency of consumption was given per day, per week and/or per month, depending on the food item. Photographs were used to visualize small, medium and large portion sizes for each food item and respondents had to choose one out of three pictures. The questionnaire was validated with a series of three $24 \mathrm{~h}$ recalls in a subsample of ninety-seven women at 14-18 weeks of gestation. Overall, the level of agreement between the FFQ and the $24 \mathrm{~h}$ recalls was satisfactory, and significant correlations were found for major food 
groups (ranging from $0 \cdot 22$ for pulses to 0.75 for meat and meat products) and nutrients (ranging from $0 \cdot 13$ for vitamin D to 0.47 for folate).

To estimate the intake of each food item in grams, portion sizes were multiplied with daily frequencies. For complex items (such as mixed dishes), standard recipes were used when available; otherwise new recipes that matched the description of the food were created and the intake of foods from different items was aggregated. Individual portion sizes and recipes were used to calculate nutrient intake on the basis of the UK food tables (McCance \& Widdowson's The Composition of Foods). The food consumption data were analysed by a software program developed at the Department of Applied Information Technology and Multimedia (TEI-Crete, Heraklion, Crete, Greece).

\section{Potential confounders}

Potential confounders included demographic, socioeconomic, lifestyle and pregnancy characteristics that have an established or potential association with dietary patterns during pregnancy or PPD or both, including: age; ethnic origin (Greek/non-Greek); education (low: $\leq 6$ years of school, medium: $>6$ years of school but $\leq 12$ years, high: university or technical college degree); parity (primiparous/multiparous); number of other children (zero; one; and two or more); housing tenure (owned/rented); house crowding (one or less, more than one person per room in the house); smoking during pregnancy (yes/no); alcohol consumption during pregnancy $(\mathrm{g} / \mathrm{d})$; physical activity during pregnancy (yes/no); maternal pre-pregnancy BMI $\left(\mathrm{kg} / \mathrm{m}^{2}\right)$; having a miscarriage or child with congenital malformation in previous pregnancies (yes/no); hospitalization during pregnancy (yes/no); gestational diabetes (yes/no); pre-eclampsia (yes/no); thyroid disorders in current pregnancy (yes/no); uterus contractions in current pregnancy (yes/no); PPD in previous pregnancies (yes/no); and total energy intake during pregnancy $(\mathrm{kJ}(\mathrm{kcal}) / \mathrm{d})$.

\section{Statistical analysis}

Principal component analysis (PCA) was used to identify dietary patterns using the women's daily intake (in $\mathrm{g}$ ) of each of the seventeen food groups as input. PCA reduces the data by forming linear combinations of the originally observed variables, thereby grouping together correlated variables, which in turn identifies any underlying dimensions in the data. The number of components that best represented the data was chosen on the basis of the screeplots and eigenvalues of the principal components, as well as on the basis of the interpretability of factor loadings. Items with loadings of $<0 \cdot 3$ on all factors were not retained, allowing for a clearer factor set. Factor scores were calculated for each person in each pattern in terms of how closely they fit the pattern. Factor scores were computed by weighting each factor loading by the factor's eigenvalue, multiplying these weights with the participant's corresponding food group intake, and summing these products.

Bivariate associations between dependent and independent variables were studied using Pearson's $\chi^{2}$ test for categorical variables. The EPDS score failed the normality test; hence, non-parametric tests were applied (Spearman's correlation, Kruskal-Wallis and Mann-Whitney tests). Dietary factor scores (entered simultaneously) were the exposure variables of interest; they were categorized into tertiles and the lowest category of intake was the reference category. Multivariable log-binomial and linear regression models were further formed to estimate adjusted relative risk (RR) or $\beta$-coefficients and 95\% CI, respectively, for the association between dietary patterns during pregnancy and PPD symptoms after adjusting for confounders. Potential confounders that related to the outcomes of interest in bivariate models with a $P$ value $<0 \cdot 2$ were included in the multivariable models. All multivariable regression models were adjusted for total energy intake using the residual $\operatorname{method}^{(20)}$. To account for the possibility of residual confounding, the remaining characteristics available in this data set were then sequentially forced into parsimonious models to ensure that the estimates associated with dietary patterns during pregnancy remained unchanged. Effect modification was evaluated using the likelihood ratio test. All association testing was conducted assuming a 0.05 significance level and a two-sided alternative hypothesis.

All analyses were performed using the Statistical Package for the Social Sciences statistical software package version $17 \cdot 0$ (SPSS Inc., Chicago, IL, USA).

\section{Results}

Complete information was available for all main model variables for 529 women (Table 1). Participants were similar to non-participants in age and educational level, but the former were more likely to work at the time of the proposal. The demographic characteristics of pregnant women in relation to PPD symptoms according to EPDS are shown in Table 1 . High levels of PPD symptoms (EPDS $\geq 13$ ) were reported by seventy-four women (14\%). Those women were more likely to be younger, less educated and to have suffered from PPD in previous pregnancies $(P<0 \cdot 05)$.

Two factors were identified to describe the dietary patterns of pregnant women (Table 2). We have chosen to give each factor a label ('Western', 'health conscious'); these labels do not perfectly describe each underlying pattern but correspond to current views on health diet, and aid in the report and discussion of results. A 'Western' dietary pattern comprised mainly meat and meat products, potatoes, sugar and sweets, cereals, fats except olive oil, salty snacks, eggs, beverages and sauces. A 'health conscious' dietary pattern comprised mainly vegetables, fruit, nuts, pulses, fish and 
Table 1 Prevalence of postpartum depressive symptoms measured by EPDS according to population characteristics $(n 529)$, Rhea Birth Cohort, Crete, Greece, 2007-2009

\begin{tabular}{|c|c|c|c|c|}
\hline & \multicolumn{2}{|c|}{ EPDS } & \multicolumn{2}{|c|}{$\begin{array}{l}\text { High level of depressive symptoms } \\
\text { (EPDS } \geq 13)\end{array}$} \\
\hline & Mean & SD & Number of subjects & $\%$ \\
\hline \multicolumn{5}{|c|}{ Maternal age (years)* } \\
\hline$\leq 20$ & $7 \cdot 2$ & $5 \cdot 51$ & 3 & $17 \cdot 6$ \\
\hline $21-30$ & $6 \cdot 9$ & $4 \cdot 99$ & 38 & $15 \cdot 1$ \\
\hline$>30$ & $5 \cdot 7$ & $4 \cdot 16$ & 33 & $13 \cdot 4$ \\
\hline \multicolumn{5}{|l|}{ Maternal origin } \\
\hline Greek & $6 \cdot 1$ & $4 \cdot 62$ & 4 & $11 \cdot 8$ \\
\hline Non-Greek & $6 \cdot 8$ & $5 \cdot 09$ & 70 & $14 \cdot 2$ \\
\hline \multicolumn{5}{|c|}{ Maternal education* } \\
\hline Low & $7 \cdot 5$ & 4.99 & 14 & $15 \cdot 4$ \\
\hline Medium & $6 \cdot 9$ & $5 \cdot 11$ & 43 & $15 \cdot 6$ \\
\hline High & $6 \cdot 2$ & $4 \cdot 97$ & 17 & $10 \cdot 5$ \\
\hline \multicolumn{5}{|l|}{ House tenure } \\
\hline Owned & $6 \cdot 7$ & $4 \cdot 97$ & 49 & $12 \cdot 8$ \\
\hline Rented & $7 \cdot 2$ & $5 \cdot 34$ & 25 & $18 \cdot 0$ \\
\hline \multicolumn{5}{|l|}{ Parity } \\
\hline Multiparous & $6 \cdot 7$ & $5 \cdot 12$ & 38 & $12 \cdot 6$ \\
\hline Primiparous & $7 \cdot 0$ & 4.99 & 36 & $15 \cdot 9$ \\
\hline \multicolumn{5}{|c|}{ Smoking during pregnancy } \\
\hline No & $6 \cdot 7$ & 4.99 & 45 & $12 \cdot 7$ \\
\hline Yes & $7 \cdot 1$ & $5 \cdot 48$ & 29 & $16 \cdot 6$ \\
\hline \multicolumn{5}{|c|}{ Physical activity during pregnancy } \\
\hline No & $6 \cdot 4$ & $5 \cdot 36$ & 67 & $16 \cdot 3$ \\
\hline Yes & $6 \cdot 8$ & $5 \cdot 03$ & 7 & $13 \cdot 8$ \\
\hline \multicolumn{5}{|l|}{ Miscarriage } \\
\hline No & $6 \cdot 8$ & $5 \cdot 13$ & 53 & $14 \cdot 2$ \\
\hline Miscarriage & $7 \cdot 1$ & $4 \cdot 89$ & 13 & $12 \cdot 6$ \\
\hline Abortion & $6 \cdot 3$ & $4 \cdot 89$ & 8 & $15 \cdot 7$ \\
\hline \multicolumn{5}{|c|}{ Hospitalization during pregnancy } \\
\hline No & $6 \cdot 7$ & 4.92 & 67 & $13 \cdot 4$ \\
\hline Yes & $8 \cdot 4$ & $6 \cdot 79$ & 7 & $23 \cdot 3$ \\
\hline \multicolumn{5}{|c|}{ Depression in previous pregnancies* } \\
\hline No & $6 \cdot 7$ & $4 \cdot 97$ & 64 & $13 \cdot 2$ \\
\hline Yes & $8 \cdot 1$ & $5 \cdot 82$ & 10 & $22 \cdot 7$ \\
\hline
\end{tabular}

EPDS, Edinburg Postpartum Depression Scale. ${ }^{\star} P<0.05$.

seafood, olive oil and dairy products. The eigenvalues were 3.6 and 1.6 for the 'Western' and 'health conscious' factors, respectively. Collectively, these factors explained $38 \%$ of the variability within the sample.

Associations between maternal intake of different food groups (below or equal $v$. above median) during pregnancy and high levels of PPD symptoms are presented in Table 3. Olive oil intake $>40 \mathrm{~g} / \mathrm{d}$ was inversely associated with high levels of PPD symptoms (OR $=0 \cdot 57,95 \% \mathrm{CI}$ $0 \cdot 35,0 \cdot 94)$. Increased intake of sugar products $(>29 \mathrm{~g} / \mathrm{d})$ showed a positive association with PPD symptoms $(\mathrm{OR}=$ $1 \cdot 66,95 \%$ CI $1 \cdot 11,2 \cdot 73$ ).

Analyses having the EPDS score as a continuous outcome variable and the two dietary patterns simultaneously as exposure variables revealed that high adherence to a 'health conscious' pattern was associated with lower EPDS scores (highest $v$. lowest tertile: $\beta$-coefficient $=$ $-1.75, \quad P=0.02$ ) after adjustment for maternal age, education, parity, house tenure, depression in previous pregnancies and total energy intake during pregnancy (Table 4).
Analyses conducted with categorical PPD symptoms as an outcome estimated that women in the second $(\mathrm{RR}=$ $0 \cdot 52,95 \%$ CI $0 \cdot 30,0.92)$ or third tertile $(\mathrm{RR}=0.51,95 \%$ CI $0 \cdot 25,1 \cdot 05)$ of the 'health conscious' dietary pattern were about $50 \%$ less likely to have high levels of PPD symptoms (EPDS $\geq 13$ ) compared with those in the lowest tertile after adjustment for maternal age, education, parity, house tenure, depression in previous pregnancies and total energy intake during pregnancy (Table 4).

To evaluate the possibility of reverse causation by women who developed depressive symptoms during pregnancy, we performed additional analyses excluding women with a diagnosis of depression during pregnancy ( $n$ 19), and the results remained essentially the same as those from the original analysis. (i) EPDS as continuous variable: second $v$. lowest tertile: adjusted $\beta$-coefficient $=$ $-1 \cdot 28,95 \% \mathrm{CI}-2 \cdot 43,-0 \cdot 12$ and highest $v$. lowest tertile: adjusted $\beta$-coefficient $=-1 \cdot 60,95 \%$ CI $-3 \cdot 11,-0 \cdot 09$; (ii) EPDS as a categorical variable: second $v$. lowest tertile: adjusted $\mathrm{RR}=0.53,95 \% \mathrm{CI} 0 \cdot 28,1 \cdot 00$ and highest $v$. lowest tertile: adjusted $\mathrm{RR}=0 \cdot 57,95 \% \mathrm{CI} 0 \cdot 32,1 \cdot 44$. 
Table 2 Factor loading of various food groups in the two principal dietary components ( $n$ 529), Rhea Birth Cohort, Crete, Greece, 2007-2009

\begin{tabular}{lrr}
\hline & \multicolumn{2}{c}{ Factor loadingst } \\
\cline { 2 - 3 } Food group & Western & Health conscious \\
\hline Meat and meat products & $\mathbf{0 . 5 6 1}$ & 0.048 \\
Potatoes and other starchy roots & $\mathbf{0 . 5 8 8}$ & 0.060 \\
Sugar, sugar preserves and confectionery & $\mathbf{0 . 5 7 2}$ & 0.084 \\
Cereals and cereal products & $\mathbf{0 . 5 6 5}$ & 0.370 \\
Fats, except olive oil & $\mathbf{0 . 4 9 4}$ & 0.311 \\
Eggs & $\mathbf{0 . 4 6 8}$ & 0.279 \\
Non-alcoholic beverages & $\mathbf{0 . 4 7 8}$ & $-0 \cdot 103$ \\
Alcoholic beverages & $\mathbf{0 . 3 9 3}$ & 0.050 \\
Salty snacks & $\mathbf{0 . 5 3 7}$ & -0.029 \\
Sauces & $\mathbf{0 . 6 2 2}$ & $0 \cdot 114$ \\
Vegetables & $0 \cdot 189$ & $\mathbf{0 . 7 0 2}$ \\
Fruit & 0.073 & $\mathbf{0 . 5 1 2}$ \\
Pulses & -0.036 & $\mathbf{0 . 5 0 0}$ \\
Olive oil & 0.147 & $\mathbf{0 . 5 8 1}$ \\
Fish and seafood & -0.004 & $\mathbf{0 . 4 9 0}$ \\
Milk and milk products & -0.028 & $\mathbf{0 . 4 3 5}$ \\
Nuts & 0.189 & $\mathbf{0 . 4 1 6}$ \\
\hline
\end{tabular}

tValues $>0.3$ are indicated in bold.

Table 3 Association between maternal intake of different food groups during pregnancy and high levels of postpartum depressive symptoms ( $n$ 529), Rhea Birth Cohort, Crete, Greece, 2007-2009

\begin{tabular}{|c|c|c|c|}
\hline \multicolumn{2}{|c|}{ Maternal food intake during pregnancy } & \multicolumn{2}{|c|}{ High levels of postpartum depressive symptoms } \\
\hline Food group & Median (g/d) & OR $\ddagger$ & $95 \% \mathrm{Cl}$ \\
\hline Meat and meat products & $>80 \cdot 60$ & $1 \cdot 28$ & $0 \cdot 61,1 \cdot 88$ \\
\hline Potatoes and other starchy roots & $>53 \cdot 17$ & $1 \cdot 02$ & $0 \cdot 62,1 \cdot 66$ \\
\hline Sugar, sugar preserves and confectionery & $>28.55$ & $1 \cdot 66^{\star}$ & $1 \cdot 11,2 \cdot 73$ \\
\hline Cereals and cereal products & $>137 \cdot 76$ & $1 \cdot 07$ & $0.52,1.67$ \\
\hline Fats, except olive oil & $>6.64$ & 0.73 & $0 \cdot 44,1 \cdot 22$ \\
\hline Eggs & $>12 \cdot 60$ & $1 \cdot 11$ & $0.68,1 \cdot 80$ \\
\hline Non-alcoholic beverages & $>163 \cdot 34$ & 1.05 & $0.54,1 \cdot 83$ \\
\hline Alcoholic beverages & $>10 \cdot 70$ & 1.92 & $0.56,7.94$ \\
\hline Salty snacks & $>2.64$ & $1 \cdot 16$ & $0.27,2.05$ \\
\hline Sauces & $>1 \cdot 78$ & 1.53 & $0 \cdot 82,2 \cdot 55$ \\
\hline Vegetables & $>212 \cdot 06$ & 0.85 & $0.51,1.47$ \\
\hline Fruit & $>408 \cdot 44$ & $0 \cdot 81$ & $0 \cdot 49,1 \cdot 32$ \\
\hline Pulses & $>8.09$ & $1 \cdot 02$ & $0 \cdot 67,1 \cdot 79$ \\
\hline Olive oil & $>40 \cdot 70$ & $0 \cdot 57^{*}$ & $0.35,0.94$ \\
\hline Fish and seafood & $>19 \cdot 60$ & 0.89 & $0.51,1 \cdot 61$ \\
\hline Milk and milk products & $>335 \cdot 71$ & 0.96 & $0.60,1.55$ \\
\hline Nuts & $>0.56$ & $1 \cdot 08$ & $0 \cdot 69,1 \cdot 75$ \\
\hline
\end{tabular}

EPDS, Edinburg Postpartum Depression Scale.

${ }^{*} \mathrm{OR}$ and $95 \% \mathrm{Cl}$ significant at $P<0.05$.

tHigh levels of postpartum depressive symptoms as measured by EPDS $\geq 13$.

$\ddagger$ All models adjusted for: maternal age, maternal education, parity, house tenure, depression in previous pregnancies and total energy intake during pregnancy. The reference group included pregnant women with consumption below or equal to median for all food groups.

\section{Discussion}

The study has shown that women who follow a 'healthy' diet during pregnancy comprising vegetables, fruit, nuts, pulses, fish and seafood, olive oil and dairy products have a reduced risk of depressive symptoms postpartum. Associations were apparent after adjustments for a wide range of possible confounding demographic, socio-economic, lifestyle and pregnancy-related variables. To our knowledge, the present study is the first one to examine prospectively, in a general population, the relationship of dietary patterns during pregnancy with PPD symptoms and risk as assessed by the EPDS, an established and widely used screening tool for PPD with high specificity and sensitivity ${ }^{(17)}$.

The evaluation of single nutrients in biochemical pathways that influence mood may be an oversimplification of a complex biochemical process. However, dietary patterns account for cumulative and interactive effects among nutrients, reflect real-world dietary preferences and may be particularly suitable for analysis in PPD epidemiology in which many dietary components could be related to the outcome of interest ${ }^{(21)}$. The results of the present study indicate that maternal adherence to a diet based on 
Table 4 Association between dietary patterns in pregnancy and postpartum depressive symptoms ( $n$ 529), Rhea Birth Cohort, Crete, Greece (2007-2009)

\begin{tabular}{|c|c|c|c|c|c|c|c|c|}
\hline \multirow[b]{3}{*}{ Dietary patterns } & \multicolumn{8}{|c|}{ EPDS } \\
\hline & \multicolumn{4}{|c|}{ Unadjusted } & \multicolumn{4}{|c|}{ Adjustedt } \\
\hline & $\beta$-Coefficient & $95 \% \mathrm{Cl}$ & $P$ value & $P$ for trend & $\beta$-Coefficient & $95 \% \mathrm{Cl}$ & $P$ value & $P$ for trend \\
\hline Western pattern & & & & 0.019 & & & & 0.067 \\
\hline First tertile (n 176) & Ref. & & & & Ref. & & & \\
\hline Second tertile (n 177) & 0.91 & $-0.14,1.96$ & 0.090 & & 0.96 & $-0 \cdot 17,2 \cdot 00$ & 0.097 & \\
\hline Third tertile $(n 176)$ & $1 \cdot 27$ & $0 \cdot 21,2 \cdot 32$ & 0.019 & & $1 \cdot 32$ & $-0 \cdot 19,2 \cdot 76$ & 0.072 & \\
\hline \multirow{7}{*}{$\begin{array}{l}\text { Healthy pattern } \\
\text { First tertile }(n 176) \\
\text { Second tertile }(n 177) \\
\text { Third tertile }(n \text { 176) }\end{array}$} & & & & $0 \cdot 242$ & & & & 0.022 \\
\hline & Ref. & & & & Ref. & & & \\
\hline & $-0 \cdot 80$ & $-1 \cdot 86,0 \cdot 25$ & $0 \cdot 136$ & & $-1 \cdot 13$ & $-2 \cdot 25,0 \cdot 00$ & 0.049 & \\
\hline & -0.63 & $-1 \cdot 69,-0.43$ & 0.042 & & $-1 \cdot 75$ & $-3 \cdot 22,-0 \cdot 28$ & 0.020 & \\
\hline & \multicolumn{8}{|c|}{ High levels of postpartum depressive symptomsł } \\
\hline & \multicolumn{4}{|c|}{ Unadjusted } & \multicolumn{4}{|c|}{ Adjusted } \\
\hline & $\mathrm{RR}$ & $95 \% \mathrm{Cl}$ & $P$ value & $P$ for trend & RR & $95 \% \mathrm{Cl}$ & $P$ value & $P$ for trend \\
\hline Western pattern & & & & 0.736 & & & & $0 \cdot 700$ \\
\hline First tertile (n 176) & Ref. & & & & Ref. & & & \\
\hline Second tertile ( $n$ 177) & $1 \cdot 08$ & $0 \cdot 61,1 \cdot 90$ & $0 \cdot 794$ & & $1 \cdot 10$ & $0.63,1.93$ & $0 \cdot 730$ & \\
\hline Third tertile $(n$ 176) & $1 \cdot 13$ & $0.57,2 \cdot 24$ & 0.737 & & $1 \cdot 14$ & $0.58,2 \cdot 26$ & 0.703 & \\
\hline Healthy pattern & & & & 0.069 & & & & 0.044 \\
\hline First tertile $(n$ 176) & Ref. & & & & Ref. & & & \\
\hline Second tertile ( $n$ 177) & 0.54 & $0.31,0.95$ & 0.032 & & 0.52 & $0.30,0.92$ & 0.026 & \\
\hline Third tertile $(n 176)$ & 0.56 & $0 \cdot 28,1 \cdot 12$ & $0 \cdot 100$ & & 0.51 & $0 \cdot 25,1 \cdot 05$ & 0.067 & \\
\hline
\end{tabular}

EPDS, Edinburg Postpartum Depression Scale; Ref., reference category; RR, relative risk.

tAll models adjusted for: maternal age, maternal education, parity, house tenure, depression in previous pregnancies and total energy intake during pregnancy. $\ddagger$ High levels of postpartum depressive symptoms as measured by EPDS $\geq 13$.

vegetables, fruit, pulses, nuts, dairy products, fish and olive oil during pregnancy has a protective effect against PPD. These results are concordant with the only other study that evaluated associations of dietary patterns with depression and anxiety in non-pregnant women ${ }^{(13)}$, and with the prospective 'SUN' (Seguimento Universidad de Navarra) project which showed that a high adherence to a Mediterranean diet may prevent the development of depressive disorders in adults ${ }^{(23,24)}$. All the food groups included in the 'health conscious' pattern are rich in several antioxidant compounds, e.g. vitamins C, E, carotenoids, Se and flavonoids, high exposure to which protects from the effects of oxidative stress. Indeed, the latter appears to be heavily implicated in depression, since a number of oxidative stress-related disturbances have been reported in depressed patients. These include oxidative damage in erythrocytic membranes; elevated lipid peroxidation products; oxidative DNA damage; reduced serum vitamins $\mathrm{C}$ and $\mathrm{E}$; increased concentrations of the endogenous inhibitor of endothelial NO synthase asymmetric dimethylarginine; and decreased $\mathrm{NO}^{(22)}$. Therefore, it is plausible to propose that the 'healthy' food pattern in our study may exert its protective effects by correcting several depression-related oxidative markers in the cortex.

With regard to fish oils, which were also part of our healthy food pattern, a growing body of research indicates their protective role in depression, attributable to their high content of $n$-3 PUFA EPA and DHA ${ }^{(11)}$. Indeed, epidemiological and intervention studies have revealed that low $n-3$ intake is associated with PPD $^{(10,25)}$, whereas a metaanalysis of twenty-three ecological studies revealed that high DHA levels in breast milk and higher seafood consumption were predictive of lower PPD rates ${ }^{(26)}$. There are only few studies on the role of other nutrients in the development of PPD and the evidence is conflicting ${ }^{(27-30)}$.

The olive oil-rich Mediterranean diet has been associated with reduced risk for Alzheimer's disease ${ }^{(31,32)}$, which is more prevalent in women. It is interesting that a significant risk factor of Alzheimer's disease is a life history of stress and depression ${ }^{(33,34)}$, which, in women, often begins with an episode of PPD ${ }^{(35)}$. To our knowledge, ours is the first study that shows a protective effect of olive oil intake $(>40 \mathrm{~g} / \mathrm{d})$ during pregnancy on PPD. Further research is required to extend the possible protective effects of olive oil in risk of depression and other affective disorders in general. Olive oil is unique in the sense that it is the only vegetable oil containing high sources of MUFA and specific antioxidants. Its high concentration of oleic acid renders olive oil resistant to lipid peroxidation ${ }^{(36,37)}$. Moreover, olive oil has a high concentration in $\alpha$-tocopherol (the most antioxidant form of vitamin E), carotenoids (such as xanthophyll and lycopene with antioxidant properties) and polyphenols with known antioxidant capacity (hydroxytyrosol and oleuropein) ${ }^{(38)}$. On account of these natural antioxidant ingredients, olive oil may potently inhibit the oxidative stress, free radical formation and neurotoxicity associated with depressive symptoms ${ }^{(39)}$. 


\section{Strengths and limitations}

The strengths of the present study include the populationbased prospective design, reasonable numbers of women with PPD symptoms and detailed data for dietary intake during pregnancy. The study population included women from the follow-up of a birth cohort, giving us the opportunity to account for the effect of exposures during pregnancy and early life measured prospectively within the cohort. The exclusion of women diagnosed with psychiatric disorders before pregnancy, as well as adjustment for several sociodemographic variables, reduced the likelihood of confounding. Participants were unaware of the hypothesis being tested; therefore, misclassification of intake estimated by the FFQ is likely to be random with respect to depressive symptoms.

The assessment of dietary intake in pregnant women is complicated because of various factors depending on the period of pregnancy. Appetite fluctuations may influence diet reports and partly explain poor correlations between dietary measurements. In the present analysis, there were specific questions on nausea symptoms during pregnancy and only $8 \%$ of the study population reported nausea symptoms at the time of the interview. A sensitivity analysis excluding women with nausea symptoms at the time of the interview yielded essentially the same results (data not shown).

There are several limitations in the present study. We assessed postpartum depressive symptomatology with the self-administered EPDS, rather than definite cases of PPD based on clinician-administered structured diagnostic interview. However, this an epidemiological study assessing risk of PPD, and EPDS is an established and widely used screening tool for PPD with high specificity and sensitivity ${ }^{(17,40)}$. Another possible limitation is the low participation rate $(57 \%)$. This limitation, however, could have affected the description of dietary patterns in a population but not the direction of the association between a healthy diet and PPD found in our study. There were no significant differences regarding sociodemographic characteristics between the women who participated in the study and those who refused to do so, except for working status. Because this variable was not related to either dietary patterns obtained by women or to PPD symptoms, it is unlikely that selection bias could have had a significant effect on our results. We have considered reports of women on diet during pregnancy; therefore, information bias could have occurred. However, studies on nutrition during pregnancy suggest that food frequency methods produce both valid and reproducible estimates of the dietary intakes in pregnant women $^{(41)}$. Although we incorporated extensive information on potential social and environmental factors that are associated with the risk of PPD, we acknowledge that there may be other factors linked with both depression and diet during pregnancy that could explain this association.
In conclusion, the present study suggests that a diet during pregnancy that is based on vegetables, fruit, pulses, nuts, dairy products, fish and olive oil may protect against postpartum depressive symptoms. Future longitudinal studies and trials are needed to confirm these findings and better understand the complex underlying processes. Given that (i) the prevalence and direct and indirect costs of PPD and affective disorders are high and (ii) diet is a modifiable environmental exposure, developing preventive mental health-care strategies based on specific dietary improvements during pregnancy could be of great benefit for public health.

\section{Acknowledgements}

The present study was partly supported by the EU Integrated Project NewGeneris, 6th Framework Programme (contract no. FOOD-CT-2005-016320). The authors have no conflict of interest to declare. None of the authors has any commercial or financial involvements that might present an appearance of a conflict of interest in connection with the submitted manuscript. L.C. - the field study coordinator participated in the study conception and design, the statistical analysis and wrote the paper; V.M., K.S. and I.A. contributed to the design of the study and drafting of the manuscript; T.R., V.G. and M.V. participated in the analysis and interpretation of the results and in drafting of the manuscript; A.K., P.B. and M.K. conceived the study, participated in its design and helped to draft the manuscript and to revise it critically. All authors contributed substantially to the study and have personally reviewed and approved the submitted manuscript.

\section{References}

1. Pearlstein T, Howard M, Salisbury A et al. (2009) Postpartum depression. Am J Obstet Gynecol 200, 357-364.

2. Gavin NI, Gaynes BN, Lohr KN et al. (2005) Perinatal depression: a systematic review of prevalence and incidence. Obstet Gynecol 106, 1071-1083.

3. Wisner KL, Moses-Kolko EL \& Sit DK (2010) Postpartum depression: a disorder in search of a definition. Arch Womens Ment Health 13, 37-40.

4. Dietz PM, Williams SB, Callaghan WM et al. (2007) Clinically identified maternal depression before, during, and after pregnancies ending in live births. Am J Psychiatry 164, 1515-1520.

5. Giakoumaki O, Vasilaki K, Lili L et al. (2009) The role of maternal anxiety in the early postpartum period: screening for anxiety and depressive symptomatology in Greece. J Psychosom Obstet Gynaecol 30, 21-28.

6. Gonidakis F, Rabavilas AD, Varsou E et al. (2008) A 6-month study of postpartum depression and related factors in Athens Greece. Compr Psychiatry 49, 275-282.

7. Vivilaki VG, Dafermos V, Kogevinas M et al. (2009) The Edinburgh Postnatal Depression Scale: translation and validation for a Greek sample. BMC Public Health 9, 329.

8. Gunlicks ML \& Weissman MM (2008) Change in child psychopathology with improvement in parental depression: a 
systematic review. J Am Acad Child Adolesc Psychiatry $\mathbf{4 7}$, 379-389.

9. Weissman MM, Pilowsky DJ, Wickramaratne PJ et al. (2006) Remissions in maternal depression and child psychopatholOgy: a STAR*D-child report. JAMA 295, 1389-1398.

10. Leung BM \& Kaplan BJ (2009) Perinatal depression: prevalence, risks, and the nutrition link - a review of the literature. J Am Diet Assoc 109, 1566-1575.

11. Freeman MP (2006) Omega-3 fatty acids and perinatal depression: a review of the literature and recommendations for future research. Prostaglandins Leukot Essent Fatty Acids 75, 291-297.

12. Jacobs DR Jr \& Steffen LM (2003) Nutrients, foods, and dietary patterns as exposures in research: a framework for food synergy. Am J Clin Nutr 78, Suppl. 3, 508S-513S.

13. Jacka FN, Pasco JA, Mykletun A et al. (2010) Association of Western and traditional diets with depression and anxiety in women. Am J Psychiatry 167, 305-311.

14. Chatzi L, Plana E, Daraki V et al. (2009) Metabolic syndrome in early pregnancy and risk of preterm birth. Am J Epidemiol 170, 829-836.

15. Davey GK, Spencer EA, Appleby PN et al. (2003) EPICOxford: lifestyle characteristics and nutrient intakes in a cohort of 33883 meat-eaters and 31546 non meat-eaters in the UK. Public Health Nutr 6, 259-269.

16. Cox JL, Holden JM \& Sagovsky R (1987) Detection of postnatal depression. Development of the 10-item Edinburgh Postnatal Depression Scale. Br J Psychiatry 150, 782-786.

17. Harris B, Huckle P, Thomas R et al. (1989) The use of rating scales to identify post-natal depression. Br J Psychiatry 154, 813-817.

18. Gibson J, McKenzie-McHarg K, Shakespeare J et al. (2009) A systematic review of studies validating the Edinburgh Postnatal Depression Scale in antepartum and postpartum women. Acta Psychiatr Scand 119, 350-364.

19. Matthey S, Henshaw C, Elliott S et al. (2006) Variability in use of cut-off scores and formats on the Edinburgh Postnatal Depression Scale: implications for clinical and research practice. Arch Womens Ment Health 9, 309-315.

20. Willet WC \& Stampfer MJ (1986) Total energy intake: implications for epidemiologic analyses. Am J Epidemiol 124, 17-27.

21. Hu FB (2002) Dietary pattern analysis: a new direction in nutritional epidemiology. Curr Opin Lipidol 13, 3-9.

22. Ng F, Berk M, Dean O et al. (2008) Oxidative stress in psychiatric disorders: evidence base and therapeutic implications. Int J Neuropsychopharmacol 11, 851-876.

23. Sanchez-Villegas A, Delgado-Rodriguez M, Alonso A et al. (2009) Association of the Mediterranean dietary pattern with the incidence of depression: the Seguimiento Universidad de Navarra/University of Navarra follow-up (SUN) cohort. Arch Gen Psychiatry 66, 1090-1098.

24. Sanchez-Villegas A, Henriquez P, Bes-Rastrollo M et al. (2006) Mediterranean diet and depression. Public Health Nutr 9, 1104-1109.

25. Rees AM, Austin MP \& Parker G (2005) Role of omega-3 fatty acids as a treatment for depression in the perinatal period. Aust N Z J Psychiatry 39, 274-280.
26. Hibbeln JR (2002) Seafood consumption, the DHA content of mothers' milk and prevalence rates of postpartum depression: a cross-national, ecological analysis. J Affect Disord 69, 15-29.

27. Beard JL, Hendricks MK, Perez EM et al. (2005) Maternal iron deficiency anemia affects postpartum emotions and cognition. J Nutr 135, 267-272.

28. Miyake Y, Sasaki S, Tanaka K et al. (2006) Dietary folate and vitamins B12, B6, and B2 intake and the risk of postpartum depression in Japan: the Osaka Maternal and Child Health Study. J Affect Disord 96, 133-138.

29. Miyake Y, Sasaki S, Yokoyama T et al. (2006) Risk of postpartum depression in relation to dietary fish and fat intake in Japan: the Osaka Maternal and Child Health Study. Psychol Med 36, 1727-1735.

30. Wojcik J, Dudek D, Schlegel-Zawadzka M et al. (2006) Antepartum/postpartum depressive symptoms and serum zinc and magnesium levels. Pharmacol Rep 58, 571-576.

31. Luchsinger JA \& Mayeux R (2004) Dietary factors and Alzheimer's disease. Lancet Neurol 3, 579-587.

32. Scarmeas N, Luchsinger JA, Schupf N et al. (2009) Physical activity, diet, and risk of Alzheimer disease. JAMA 302, 627-637.

33. Bao AM, Meynen G \& Swaab DF (2008) The stress system in depression and neurodegeneration: focus on the human hypothalamus. Brain Res Rev 57, 531-553.

34. Simard M, Hudon C \& van Reekum R (2009) Psychological distress and risk for dementia. Curr Psychiatry Rep 11, $41-47$.

35. Robling SA, Paykel ES, Dunn VJ et al. (2000) Long-term outcome of severe puerperal psychiatric illness: a 23 year follow-up study. Psychol Med 30, 1263-1271.

36. Mensink RP \& Katan MB (1989) Effect of a diet enriched with monounsaturated or polyunsaturated fatty acids on levels of low-density and high-density lipoprotein cholesterol in healthy women and men. $N$ Engl J Med 321, 436-441.

37. Kasdallah-Grissa A, Nakbi A, Koubaa N et al. (2008) Dietary virgin olive oil protects against lipid peroxidation and improves antioxidant status in the liver of rats chronically exposed to ethanol. Nutr Res 28, 472-479.

38. Fabiani R, Rosignoli P, De Bartolomeo A et al. (2008) Oxidative DNA damage is prevented by extracts of olive oil, hydroxytyrosol, and other olive phenolic compounds in human blood mononuclear cells and HL60 cells. J Nutr 138, 1411-1416.

39. Paiva-Martins F, Fernandes J, Rocha S et al. (2009) Effects of olive oil polyphenols on erythrocyte oxidative damage. Mol Nutr Food Res 53, 609-616.

40. Thompson WM, Harris B, Lazarus J et al. (1998) A comparison of the performance of rating scales used in the diagnosis of postnatal depression. Acta Psychiatr Scand 98, 224-227.

41. Meltzer HM, Brantsaeter AL, Ydersbond TA et al. (2008) Methodological challenges when monitoring the diet of pregnant women in a large study: experiences from the Norwegian Mother and Child Cohort Study (MoBa). Matern Child Nutr 4, 14-27. 